\title{
Microscopic Measurement of Photon Echo Formation in Groups of Individual Excitonic Transitions
}

\author{
Wolfgang Langbein \\ Department of Physics and Astronomy, Cardiff University, Cardiff CF24 3YB, United Kingdom* \\ Brian Patton \\ Experimentelle Physik IIb, Universität Dortmund, Otto-Hahn-Str. 4, 44221 Dortmund, Germany
}

(Received 3 February 2005; published 30 June 2005)

\begin{abstract}
The third-order polarization emitted from groups of individual localized excitonic transitions after pulsed optical excitation is measured. We observe the evolution of the nonlinear response from the case of a free polarization decay for a single transition, to that of a photon echo for many transitions. The echo is shown to arise from the mutual rephasing of the emission from individual transitions.
\end{abstract}

DOI: 10.1103/PhysRevLett.95.017403

PACS numbers: 78.47.+p, 42.65.Re, 78.66.Fd, 78.67.Hc

Nonlinear optical spectroscopy is a powerful technique when investigating the electronic structure and dynamics of matter. Specifically, the third-order nonlinearity probed in four-wave mixing (FWM), spectral hole burning, and pump-probe experiments can be used to determine homogeneous line shapes of inhomogeneously broadened transitions. This was first employed for nuclear spin transitions excited by radio frequencies, which show spin echoes [1]. The availability of ultrafast laser pulses allowed the echo technique to be extended to optical frequencies [2,3]. More recent experiments have seen the effect of inhomogeneous broadening circumvented by measuring on individual systems, such as single atoms in traps, single molecules on surfaces, or single localized exciton states in semiconductor quantum dots. This is currently only possible in the optical frequency range in which highly sensitive detectors are available. In such experiments, the measurement time has to be much longer than the dephasing time in order to collect a sufficient number of photons. During this time, fluctuations in the environment lead to slow spectral diffusion of the frequency of the investigated transitions (e.g., by the Stark effect), resulting in an inhomogeneous broadening in the measurement due to the time ensemble [4]. As a result, the typical linewidth of semiconductor quantum dots at low temperatures measured in single-dot photoluminescence is $10-1000 \mu \mathrm{eV}$ [5-8], while the homogeneous linewidth determined in photon echo experiments is about $1 \mu \mathrm{eV}$ [9-11] and can be given by the natural linewidth, i.e., the radiative decay rate. FWM spectroscopy on individual exciton transitions can distinguish between these two broadening mechanisms. However, until now, FWM on single exciton states was only performed using continuous wave excitation with nondegenerate frequencies $[12,13]$. Therefore, the formation of the photon echo from the interference of the FWM polarizations of individual transitions has, up to now, not been observed.

In this Letter, we present transient four-wave mixing measurements on individual, localized excitons. Using a multichannel heterodyne detection, the frequency-resolved third-order polarization is measured in amplitude and phase, so that both the time- and the spectrally resolved third-order polarization can be retrieved. Analogous to the introduction of pulsed nuclear magnetic resonance spectroscopy to replace scanning techniques, the detection sensitivity is increased significantly by the multichannel detection. This enables us to investigate the evolution of the nonlinear response from the case of a free polarization decay to that of a photon echo as the number of individual transitions in an inhomogeneously broadened ensemble is increased.

The size of the investigated excitonic states is much smaller than the wavelength of the resonant light. Therefore, the emitted polarization of an individual state is essentially isotropic (apart from polarization effects), rendering the commonly used directional selection of the four-wave mixing signal useless. To discriminate the fourwave mixing signal in such a case, we have to use the phase coherence of the signal relative to the excitation pulses, which is determined by the form of the third-order polarization $P^{(3)} \propto E_{1}^{*} E_{2} E_{2}$, with the excitation electric fields $E_{1,2}$. In order to be sensitive to the phase of the emitted FWM field, we detect it via its interference with a reference field $E_{\mathrm{r}}$. Such a detection principle was used to measure pump-probe and four-wave mixing in waveguides $[14,15]$ and in planar InAs quantum dot ensembles [10]. In these investigations of large ensembles of excitonic states $(N>$ $10^{5}$ ), the FWM was emitted as a photon echo [9] of $\approx 100 \mathrm{fs}$ duration given by the inverse inhomogeneous broadening of the transition energies in the excited ensemble. Conversely, when probing individual transitions, the signal of each transition is expected to be emitted as a free polarization decay, with a decay time given by the intrinsic dephasing time of the transition, which can be many orders of magnitude longer than the ensemble photon echo. In order to efficiently measure a signal of such rich spectral complexity, a multichannel detection is needed. 
We employ a multichannel heterodyne scheme using spectrally resolved detection with a charge-coupled device (CCD), and retrieve the signal by spectral interferometry [16]. This scheme provides a simultaneous measurement of all spectral components of the signal in both amplitude and phase, allowing the determination of the signal in both frequency and time domain by Fourier transform.

A schematic overview of the experimental setup is given in Fig. 1. We start with optical excitation pulses of 0.2 ps duration at $76 \mathrm{MHz}$ repetition rate from a mode-locked Ti:sapphire laser with a center frequency $\omega_{0}$. Two pulse trains 1,2 are created by a beam splitter, frequency shifted with acousto-optical modulators (AOMs) by the radio frequencies $\Omega_{1} / 2 \pi=79 \mathrm{MHz}$ and $\Omega_{2} / 2 \pi=80 \mathrm{MHz}$, and recombined into the same spatial mode, with a relative delay time $\tau$, positive for pulse 1 leading. A frequencyunshifted reference pulse train is recombined with the pulse trains 1, 2 in such a way as to pass through the same optics, but in a slightly different direction. In this manner, passive phase stability between the excitation and the reference beams is achieved over the whole optical path. The pulses are focused onto the sample using a microscope objective of a numerical aperture $\mathrm{NA}=0.85$ mounted in a helium bath cryostat. The FWM signal is collected by the same objective, and directed into a mixing $\mathrm{AOM}$, in such a way that the diffracted beam of the signal overlaps with the reference beam reflected by the sample and vice versa. The mixed beams $a, b$ (see Fig. 1) are spectrally resolved and detected by a liquid nitrogen cooled silicon CCD, measuring their spectrally and timeresolved intensities $I_{a, b}(\omega, t)$. In this notation, the variable $\omega$ describes the optical frequencies, with a resolution given by the spectrometer $(\approx 3 \mathrm{GHz})$, while the time variable $t$ describes the low-frequency dynamics, which contains the modulation due to the frequency shifts by the AOMs [17]. The mixing AOM down-shifts the signal field $E_{\mathrm{s}}$ in frequency by $\Omega_{\mathrm{d}}$ when deflected into the reference field $E_{\mathrm{r}}$, while the reference is frequency up-shifted by $\Omega_{\mathrm{d}}$ when

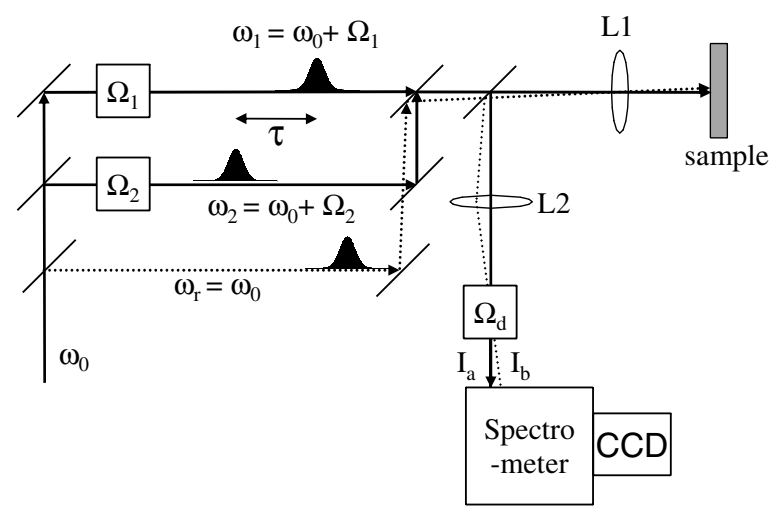

FIG. 1. Scheme of the experimental setup. Boxes: Acoustooptical modulators of the indicated frequency. L1: High numerical aperture (0.85) microscope objective. Spectrometer: Imaging spectrometer of $15 \mu \mathrm{eV}$ resolution. deflected into the signal. In this way, the interference frequency of signal and reference fields in the beams $a, b$ is shifted by $\Omega_{\mathrm{d}}$. The deflection efficiency is adjusted to $50 \%$, so that the detected intensities are given by $2 I_{a, b}(\omega, t)=\left|E_{\mathrm{r}}\right|^{2}+\left|E_{\mathrm{s}}\right|^{2} \pm 2 \Re\left(E_{\mathrm{r}} E_{\mathrm{s}}^{*} e^{i \Omega_{\mathrm{d}} t}\right)$. Since we detect $I_{a, b}(\omega, t)$ temporally integrated over the exposure time $T$ of the CCD (which is typically $50-1000 \mathrm{~ms}$ ), only the interference close to zero frequency $(\Omega<1 / T)$ is detected. To recover the interference term, we subtract the two detected intensities, yielding $I_{\mathrm{d}}(\omega)=I_{a}-I_{b}=$ $2 \int_{0}^{T} \mathfrak{R}\left(E_{\mathrm{r}} E_{\mathrm{s}}^{*} e^{i \Omega_{\mathrm{d}} t}\right) d t$.

Having measured the interference intensity $I_{\mathrm{d}}(\omega)$, we deduce the signal field in amplitude and phase by spectral interferometry [16], using the fact that the reference field is adjusted to precede the signal field in time $F\left\{\Theta(t) F^{-1}\left[I_{\mathrm{d}}(\omega)\right]\right\}=E_{\mathrm{r}}^{*}(\omega) E_{\mathrm{s}}(\omega) e^{i \Omega_{\mathrm{d}} t} \quad$ with the Heaviside function $\Theta(t)$, and the Fourier-transform operator $F$. The time range of the resulting time-resolved signal field of this technique is limited by the spectral resolution to about $100 \mathrm{ps}$, which is much smaller than the repetition period. The reference field amplitude can be determined by blocking the signal beam and measuring $I_{a, b}=\left|E_{\mathrm{r}}\right|^{2}$ in this case. The reference phase can be determined by nonlinear pulse characterization techniques, or can be calculated from the chirp introduced by the optical components in the setup. Knowing the reference field $E_{\mathrm{r}}(\omega)$, the signal field $E_{\mathrm{s}}(\omega)$ can be determined. The choice of $\Omega_{\mathrm{d}}$ selects the detected interference. For $\Omega_{\mathrm{d}}=\Omega_{1,2}$, the reflected excitation pulses 1, 2 are measured, while for $\Omega_{\mathrm{d}}=2 \Omega_{2}-\Omega_{1}$, the emitted FWM field $\propto E_{1}^{*} E_{2} E_{2}$ is measured. Higherorder nonlinearities, like six-wave mixing, can be detected analogously.

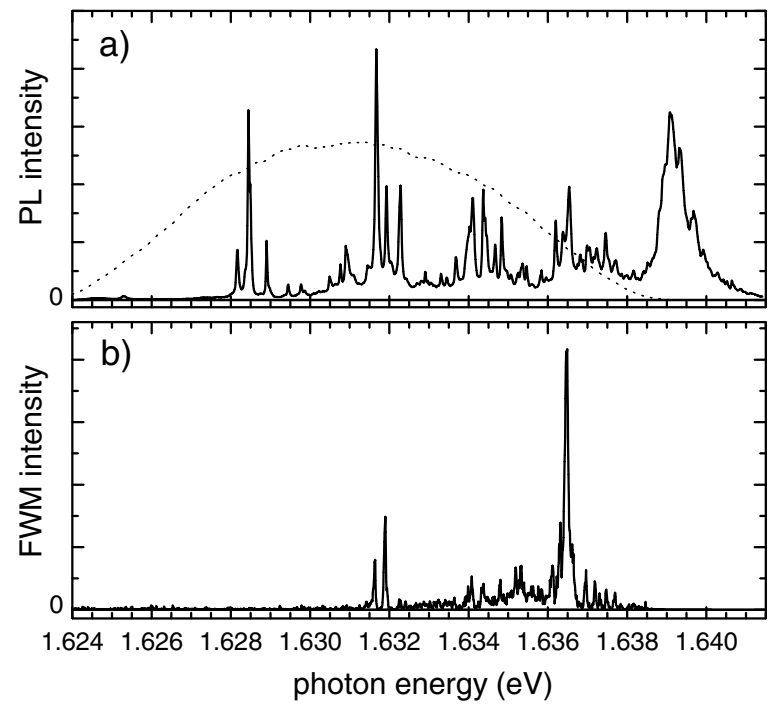

FIG. 2. (a) Confocally excited/detected PL spectrum of a $(0.5 \mu \mathrm{m})^{2}$ area of a AlAs/GaAs QW with a thickness of about $20.8 \mathrm{ML}(6 \mathrm{~nm})$. The spectrum of the excitation pulses used in the FWM experiment of (b) and Fig. 3 is shown as a dotted line. (b) Spectrally resolved FWM intensity at $\tau=1 \mathrm{ps}$. 
The investigated sample [18] consists of an AlAs/GaAs/AlAs single quantum well grown by molecular-beam epitaxy with a thickness increasing from approximately 4 to $10 \mathrm{~nm}$ over a total lateral size of $200 \mathrm{~mm}$. The growth was interrupted for $120 \mathrm{~s}$ at each interface, allowing for the formation of large monolayer islands on the growth surface. The sample was antireflection coated, and was held in a helium cryostat at a temperature of $T=5 \mathrm{~K}$. Exciting nonresonantly at $1.96 \mathrm{eV}$, focused to the diffraction limit, the confocally detected photoluminescence (PL) is shown in Fig. 2(a). In order to select individual states within the optical resolution of the experiment $(0.5 \mu \mathrm{m})$, we adjusted the fractional monolayer thickness of the quantum well (QW) to be about -0.2 ML [19], yielding very few localized exciton states in the largest monolayer (ML) thickness. This was done by monitoring the PL spectrum while moving the excitation spot along the QW thickness gradient. Individual emission lines are visible in the low-energy part of the spectrum, corresponding to individual localized excitons. Because of the diffusion of the excited carriers prior to recombination, the spatial resolution of the PL spectrum is not significantly improved by the confocal excitation, and is essentially determined by the diffraction limited intensity resolution of $0.61 \lambda / \mathrm{NA}$ in the emission imaging. The same region was investigated by the FWM technique. The laser pulse spectrum shown in Fig. 2(a) excited only the excitons localized in the lower monolayer in order to avoid large densities of excited excitons. The spectrally resolved FWM at $\tau=1$ ps is given in Fig. 2(b). It consists of several sharp resonances of 20-30 $\mu \mathrm{eV}$ FWHM. The FWM intensity is proportional to the third power of the excitation intensity, which improves the spatial resolution to $0.36 \lambda / \mathrm{NA} \approx$ $320 \mathrm{~nm}$. Only at the higher energy side of the PL emission are FWM resonances of significant strength observed. The FWM intensities of the resonances are not clearly corre-

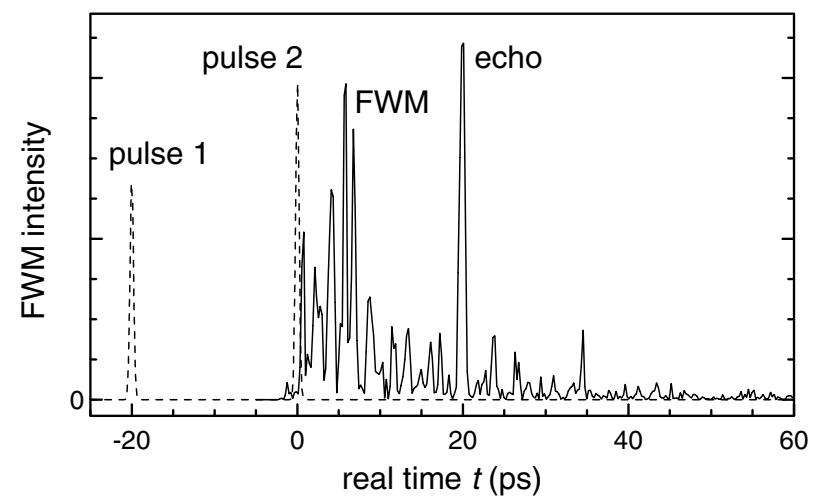

FIG. 3. Time-resolved FWM intensity from a group of exciton states localized within an $(0.5 \mu \mathrm{m})^{2}$ area. Excitation pulses 1, 2 (dashed line) and time-resolved FWM intensity (solid line) for a delay time of $\tau=20 \mathrm{ps}$ are shown. Excitation energies were 1.0 (1.9) fJ for pulse 1(2), corresponding to an intensity per pulse of $0.4(0.75) \mu \mathrm{J} / \mathrm{cm}^{2}$ at the quantum well. lated to the PL intensities. This can be understood considering the properties of the resonances determining the respective signal strength. The FWM intensity is determined only by the fourth power of the optical transition dipole moment $\mu$ of the resonance. The PL intensity instead is determined by the radiative rate, proportional to $\mu^{2}$, and also by the relaxation dynamics of excitons into the localized state. Additionally, in the PL we use nonresonant excitation, for which charged exciton emission [20], having a binding energy of about $4 \mathrm{meV}$, could be present, explaining the lower energy peaks (e.g., at $1.628 \mathrm{eV}$ ) in the PL.

The temporal sequence of the experiment, consisting of the arrival of pulse 1, pulse 2, and the subsequent emission of the FWM intensity can be measured in the experiment by choosing $\Omega_{\mathrm{d}}$ equal to $\Omega_{1}, \Omega_{2}$, or $2 \Omega_{2}-\Omega_{1}$, respectively, and is shown in Fig. 3. Pulse 1 arrives at $t=$ $-20 \mathrm{ps}$, and creates a first-order polarization that oscillates at the resonance frequencies of the system. Upon the arrival of pulse 2 at $t=0$, this polarization interferes with pulse 2 and creates a density that oscillates with the frequency $\Omega_{2}-\Omega_{1}$. The polarization that is created by pulse 2 and that is proportional to this density is the thirdorder polarization. It has the frequency $2 \Omega_{2}-\Omega_{1}$, and is the source of the self-diffracted FWM. The FWM is thus expected to start at $t=0$, in agreement with the experimental observation. Since several resonances of frequencies $\omega_{k}$ are emitting, their superposition leads to a strong temporal beating in the signal. Generally, we can express the emitted FWM field $E^{(3)}$ for $\tau>0$ for a set of $N$ twolevel systems as

$$
E^{(3)}(t, \tau)=\sum_{k=1}^{N} \mu_{k}^{4} E_{1}\left(\omega_{k}\right) E_{2}^{2}\left(\omega_{k}\right) e^{i \omega_{k}(t-\tau)-\gamma_{k}(t+\tau)}
$$

with the dipole moments of the transitions $\mu_{k}$ and the appropriately normalized excitation field amplitudes $E_{1,2}(\omega)$ of pulse 1, 2 at the frequency $\omega$. Here we assume that the pulse spectral width is much larger than the de-

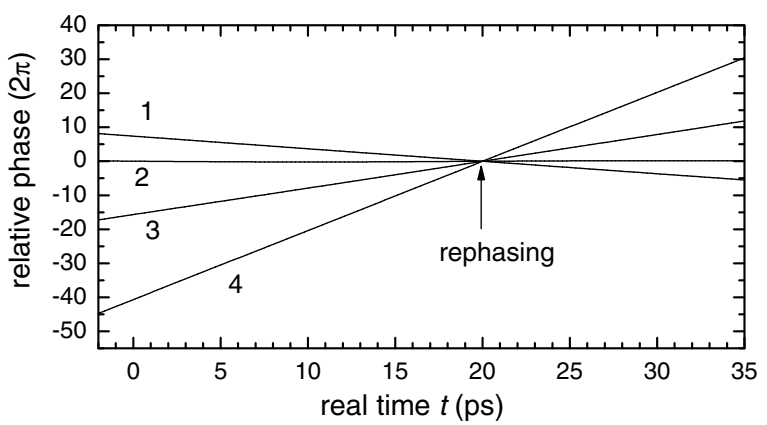

FIG. 4. Measured relative phase evolution of the FWM field of 4 individual transitions of an ensemble. The corresponding FWM spectrum is shown in the middle row of Fig. 5. The phase noise of about $0.3 \mathrm{rad}$ is not readily discernible due to the large phase scale. 

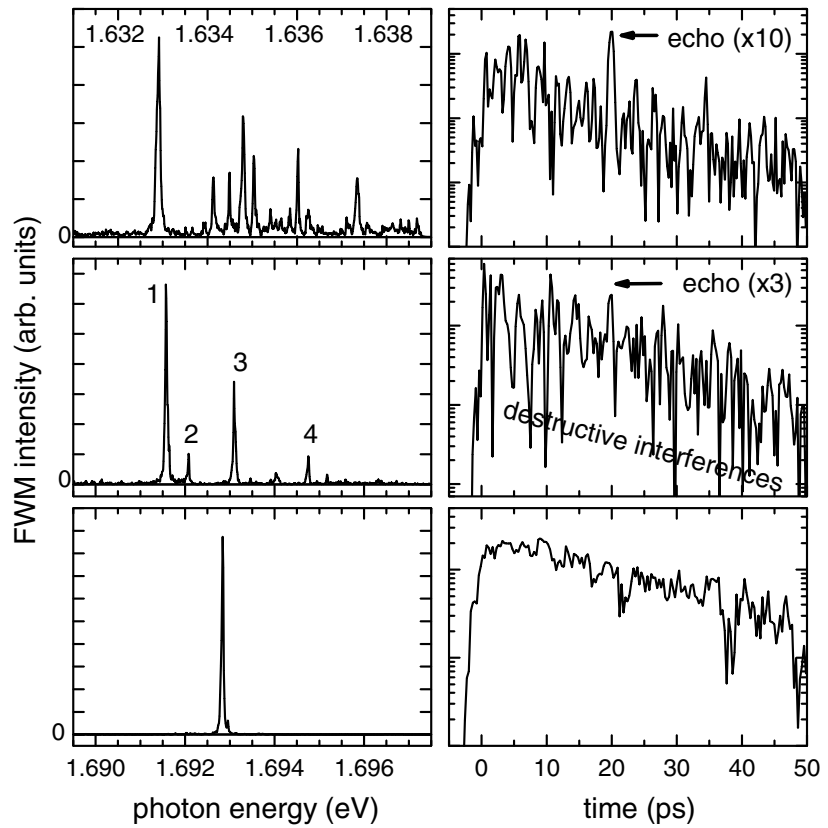

FIG. 5. FWM intensity for $\tau=20 \mathrm{ps,} \mathrm{spectrally} \mathrm{resolved} \mathrm{(left)}$ and time resolved (right), for exciton state ensembles of different size. The estimated fractional enhancement by the superradiance at the photon echo time $t=20 \mathrm{ps}$ is indicated.

phasing rates $\gamma_{k}$ of the transitions. At $t=\tau$, the FWM fields from all two-level systems are predicted to be in phase, and therefore to interfere constructively. This results in a signal amplitude $N$ times larger than the individual FWM amplitudes of a single transition. For other times, the phases are, in general, randomly distributed due to the distribution of the transition frequencies, and the enhancement is reduced to $\approx \sqrt{N}$. In the limit of a large number of systems in the ensemble, the signal at $t=\tau$ is thus far larger than at other times, and is called a photon echo. Until now, the signal away from the echo time, in the random interference time, was not observed. Here, we can see (Fig. 3) the formation of the photon echo by the constructive interference at $t=20 \mathrm{ps}$. Since we measure the phase and amplitude of the signal, we can determine the phase evolution for each resonance separately by applying a spectral filter. The result of such an analysis for 4 transitions of an ensemble is shown in Fig. 4 (the corresponding spectrally resolved FWM is given in the middle row of Fig. 5). In the measured phase evolution, the rephasing of the FWM fields from the individual transitions at the photon echo time $t=20 \mathrm{ps}$ is directly observed.

Furthermore, the fact that we can determine, in the spectral domain, the number of states contributing to the time domain FWM signal allows us, by appropriate selection of regions of the sample, to control the size of the state ensemble probed in the FWM. By systematically increasing the number of participating transitions from one to many, we can therefore follow the formation of the photon echo with increasing ensemble sizes. This evolution is shown in Fig. 5 for ensembles of about 1, 4, and 10 transitions. With increasing ensemble size $N$, the intensity enhancement in the photon echo increases roughly $\propto N$, as expected from Eq. (1). The peaks off the photon echo time are due to subsidiary constructive interferences of subgroups of states, and are prominent due to the small number of participating states.

In conclusion, we have measured the transient thirdorder polarization of individual quantum states in both amplitude and phase. The formation of the photon echo with increasing ensemble size was experimentally demonstrated and the signal was shown to vary from a free polarization decay for the single emitter to a photon echo in the ensemble limit; we have thus demonstrated experimentally the microscopic origin of the photon echo in transient four-wave mixing in a solid state system.

The sample was grown at III-V Nanolab, a joint laboratory between Research Center COM and the Niels Bohr Institute, Copenhagen University. Continued support by U. Woggon is acknowledged. This work was funded by the German Science Foundation (DFG) within the Grant No. WO477/14.

*Electronic address: LangbeinWW@cardiff.ac.uk

[1] E. L. Hahn, Phys. Rev. 80, 580 (1950).

[2] N. A. Kurnita, I. D. Abella, and S. R. Hartmann, Phys. Rev. Lett. 13, 567 (1964).

[3] M. Cho, N. F. Scherer, G. R. Fleming, and S. Mukamel, J. Chem. Phys. 96, 5618 (1992).

[4] S. Mukamel, Principles of Nonlinear Optical Spectroscopy (Oxford University, New York, 1999).

[5] M. Bayer and A. Forchel, Phys. Rev. B 65, 041308(R) (2002).

[6] K. Leosson, J. R. Jensen, J. M. Hvam, and W. Langbein, Phys. Status Solidi B 221, 49 (2000).

[7] F. Gindele, K. Hild, W. Langbein, and U. Woggon, Phys. Rev. B 60, R2157 (1999).

[8] V. Türck et al., Phys. Rev. B 61, 9944 (2000).

[9] P. Borri et al., Phys. Rev. Lett. 87, 157401 (2001).

[10] W. Langbein et al., Phys. Rev. B 70, 033301 (2004).

[11] R. Kuribayashi et al., Phys. Rev. B 57, R15084 (1998).

[12] J. Guest et al., Science 293, 2224 (2001).

[13] N. H. Bonadeo et al., Phys. Rev. Lett. 81, 2759 (1998).

[14] K. L. Hall, G. Lenz, E. P. Ippen, and G. Raybon, Opt. Lett. 17, 874 (1992).

[15] P. Borri et al., Opt. Commun. 164, 51 (1999).

[16] L. Lepetit, G. Chériaux, and M. Joffre, J. Opt. Soc. Am. B 12, 2467 (1995).

[17] This separation of time scales is possible for dephasing times shorter than the radio frequency period and the pulse repetition time.

[18] K. Leosson, J. R. Jensen, W. Langbein, and J. M. Hvam, Phys. Rev. B 61, 10322 (2000).

[19] V. Savona, W. Langbein, and G. Kocherscheidt, Phys. Status Solidi C 1, 501 (2004).

[20] J. G. Tischler, A. S. Bracker, D. Gammon, and D. Park, Phys. Rev. B 66, 081310(R) (2002). 